\title{
Dependence of LC VCO Oscillation Frequency on Bias Current
}

\author{
Ting $\mathrm{Wu}, \mathrm{Un}-\mathrm{Ku}$ Moon, and Kartikeya Mayaram \\ School of Electrical Engineering and Computer Science \\ Oregon State University, 1148 KEC, Corvallis, OR 97331
}

\begin{abstract}
LC-tuned voltage controlled oscillators (LC VCOs) are widely used in high performance phase locked loops (PLLs) and frequency synthesizers due to their high spectral purity. The oscillation frequency of an LC VCO is commonly assumed to be the resonant frequency of the LC tank. However, this is not accurate as shown in this paper. The oscillation frequency is also affected by the bias current. By applying our analysis to the Hegazi and Abidi's LC VCO, we propose a new digital trimming technique which can compensate for process variations and improve the phase noise performance.
\end{abstract}

\section{INTRODUCTION}

To sustain oscillations, an oscillator must satisfy the Barkhausen criteria. Namely, for a positive feedback system, oscillations will occur when the loop gain has a zero phase shift with a magnitude of one at the oscillation frequency. In a practical oscillator the startup loop-gain magnitude should be greater than one in order for oscillations to build up to the steady-state amplitude that is limited by other nonlinear effects.

Fig. 1(a) shows a high-level oscillator schematic, where $Y$ denotes a resonator admittance and $Y_{(-)}$denotes a negative admittance that compensates for the energy losses in the resonator.

$$
\begin{aligned}
& Y=G \dashv j B \\
& Y_{(-)}=G_{(-)}+j B_{(-)}
\end{aligned}
$$

In the steady state of an oscillator, the phase condition requires that the loop phase shift is zero. As a consequence,

$$
B_{(-)}+B=0
$$

The oscillation frequency of a LC VCO, $\omega_{\text {osc }}$, is commonly assumed to be the resonant frequency $\omega_{0}$, expressed by (4). However, this is not accurate in practical circuits [1] because of energy losses in the resonator inductor and capacitor.

$$
\omega_{O S C}=\omega_{0}=\frac{1}{\sqrt{L C}}
$$

To understand this, a simplified model of a LC oscillator is shown in Fig. 1(b), where $R_{L}$ and $R_{C}$ represent the series losses of the inductor $(L)$ and capacitor $(C)$, respectively.

The admittance of the resonator is

$$
Y=\frac{1}{j \omega L+R_{L}}+\frac{1}{1 /(j \omega C)+R_{C}}=G+j B
$$

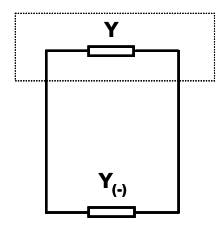

(a)

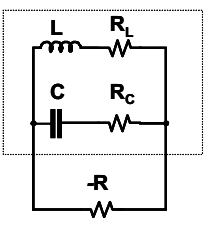

(b)
Fig. 1. (a) A general oscillator model. (b) A model of LC-tank oscillator.

where $G$ and $B$ are

$$
\begin{aligned}
& G=\frac{R_{L}}{R_{L}^{2}+\omega^{2} L^{2}}+\frac{R_{C}}{R_{C}{ }^{2}+1 /\left(\omega^{2} C^{2}\right)} \\
& B=\frac{-\omega L}{R_{L}{ }^{2}+\omega^{2} L^{2}}+\frac{1 /(\omega C)}{R_{C}{ }^{2}+1 /\left(\omega^{2} C^{2}\right)}
\end{aligned}
$$

If the LC oscillator is connected to a pure negative resistor $(-R)$, then $B_{(-)}$is zero. From the phase condition (3), $B$ must also equal to zero.

$$
B=\frac{a C}{1+1 / Q_{C}{ }^{2}}-\frac{1 / a L}{1+1 / Q_{L}{ }^{2}}=0
$$

where $Q_{C}$ and $Q_{L}$ are the quality factors of the capacitor and the inductor, respectively. These are defined by

$$
Q_{L}=\omega L / R_{L} \quad Q_{C}=1 / \omega C R_{C}
$$

Assume that both $Q_{C}$ and $Q_{L}$ are much larger than 1 . Then it can be shown that

$$
\omega^{2} L C=\left(1+\frac{1}{Q_{C}{ }^{2}}\right) /\left(1+\frac{1}{Q_{L}{ }^{2}}\right) \approx 1+\frac{1}{Q_{C}{ }^{2}}-\frac{1}{Q_{L}{ }^{2}}
$$

The relationship of the oscillation frequency $\omega_{\text {osc }}$ to the resonant frequency $\omega_{0}$ is

$$
\omega_{O S C}{ }^{2} \approx \omega_{0}^{2}\left(1-\frac{1}{Q_{L}{ }^{2}}+\frac{1}{Q_{C}{ }^{2}}\right)
$$

In other words, the oscillation frequency deviates from the resonant frequency due to the losses in the inductor and capacitor. The energy loss in the inductive branch causes the oscillation frequency to decrease, while the loss in capacitive branch causes the oscillation frequency to increase [1].

\section{BIAS CURRENT DEPENDENCE OF LC VCO}

Cross-coupled transistors are commonly used to produce a negative impedance in differential LC VCOs. Shown in Fig. 2(a) is a NMOS cross-coupled pair. 


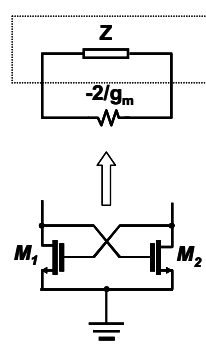

(a)

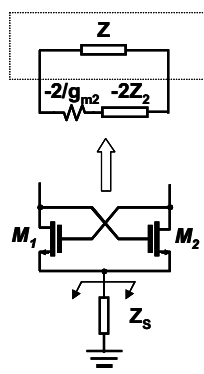

(b)
Fig. 2. Negative impedances stem from the cross-coupled transistors. (a) At DC. (b) At high frequencies.

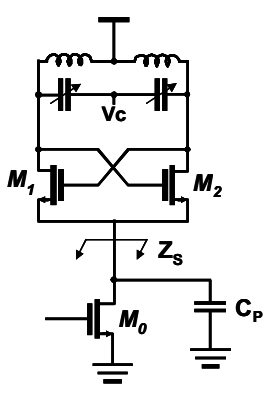

(a)

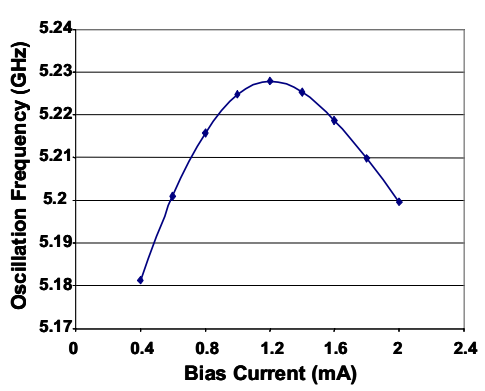

(b)
Fig. 3. (a) Schematic of a LC VCO

(b) Simulated oscillation frequency of this VCO vs. bias current.

This cross-coupled transistor pair creates a negative impedance $\left(-2 / g_{m}\right)$ across the resonator, where $g_{m}$ is the transconductance of the NMOS transistor. This is true only at low frequency. At high frequencies, an impedance $Z_{S}$ is seen by the source node of the cross-coupled transistors, as shown in Fig. 2(b). Transistor current at even harmonics (where the $2^{\text {nd }}$ harmonic component is dominant) sinks into the source impedance $Z_{S}$. It can be shown that, at the $2^{\text {nd }}$ harmonic frequency, the equivalent impedance seen by the resonator is $\left(-2 / g_{m 2}-2 Z_{2}\right)$, where $g_{m 2}$ and $Z_{2}$ denote the NMOS transconductance $g_{m}$ and the source impedance $Z_{S}$ at the $2^{\text {nd }}$ harmonic frequency.

For example, Fig. 3(a) shows the schematic of a commonly used LC VCO, where M0 is a tail current source transistor, and M1 and M2 are cross-coupled transistors. The resonator consists of inductors and varactors. As explained above, the total negative impedances associated with the cross-coupled transistors include a DC component and a $2^{\text {nd }}$ harmonic component. At the $2^{\text {nd }}$ harmonic frequency, the source impedance $Z_{S}$ is given by

$$
\left.Z_{S}\right|_{2^{\text {nd }} \text { harmonic }}=Z_{2}=1 /\left(j 2 \omega_{O S C} C_{P}\right) \approx 1 /\left(j 2 \omega_{0} C_{P}\right)=Z_{20}
$$

where $C_{P}$ denotes the total capacitance at the source node of the cross-coupled transistors, including the source capacitance of M1 and M2, the drain capacitance of M0 and the parasitic capacitance at the source node. Using this information, one can obtain

$$
B_{(-)}=\frac{-\omega_{O S C} C_{P}}{1+4 \omega_{O S C}{ }^{2} C_{P}{ }^{2} / g_{m 2}{ }^{2}}=\frac{-\omega_{O S C} C_{P}}{1+1 /\left(g_{m 2}{ }^{2}\left|Z_{2}\right|^{2}\right)} \approx \frac{-\omega_{0} C_{P}}{1+1 /\left(g_{m 2}{ }^{2}\left|Z_{20}\right|^{2}\right)}
$$

The phase condition of (3) yields

$$
B+B_{(-)}=\frac{\omega C}{1+1 / Q_{C}{ }^{2}}-\frac{1 / \omega L}{1+1 / Q_{L}{ }^{2}}+\frac{-a_{0} C_{P}}{1+1 /\left(g_{m 2}{ }^{2}\left|Z_{20}\right|^{2}\right)}=0
$$

Similar to the derivation in Section I, it can be shown that the oscillation frequency is a function of the source node capacitance $C_{P}$ and the transconductance $g_{m 2}$, as given by

$$
\omega_{\text {OSC }}{ }^{2} \approx \omega_{0}{ }^{2}\left(1-\frac{1}{Q_{L}{ }^{2}}+\frac{1}{Q_{C}{ }^{2}}+\frac{C_{P}}{C}\left(1-\frac{1}{g_{m 2}{ }^{2}\left|Z_{20}\right|^{2}}\right)\right)
$$

Therefore, the source node capacitance $C_{P}$ causes the oscillation frequency to increase. Furthermore, since $g_{m 2}$ increases with an increase in the bias current, (14) implies that the oscillation frequency of this LC VCO also increases with an increase in the bias current.

The oscillation frequency variation due to the source node capacitance $C_{P}$ can also be explained as follows. The cross-coupled transistors effectively transfer the source node capacitance as a negative capacitance that is seen by the resonator. For narrowband applications, this negative capacitor can be considered as an equivalent inductor [2]. If we define this equivalent inductor as $L_{E}$ and its quality factor as $Q_{E}$, then

$$
B_{(-)}=\frac{-\omega_{O S C} C_{P}}{1+4 \omega_{O S C}{ }^{2} C_{P}{ }^{2} / g_{m 2}{ }^{2}}=\frac{-1 / \omega_{O S C} L_{E}}{1+1 / Q_{E}{ }^{2}}
$$

from which we obtain

$$
L_{E}=1 / C_{P} \omega_{O S C}{ }^{2}, \quad Q_{E}=g_{m 2}{ }^{2} / 4 C_{P}{ }^{2} \omega_{O S C}{ }^{2}
$$

Equivalently, an additional inductor $L_{E}$ is connected in parallel with the original resonator. The total inductance is then reduced, resulting in an increased frequency of oscillation. Since $Q_{E}$ increases with an increase in $g_{m 2}$ (bias current), (10) suggests that the oscillation frequency also increases with an increase in bias current.

To verify our analysis, we have designed and simulated a LC VCO employing the topology in Fig. 3(a) in a $0.18 \mu \mathrm{m}$ CMOS technology with a $1 \mathrm{~V}$ supply voltage. The simulated oscillation frequency as a function of the bias current is shown in Fig. 3(b). As expected, for small bias currents, the oscillation frequency increases with an increase in the bias current.

When the bias current is larger than $1.2 \mathrm{~mA}$ the oscillation frequency drops with an increase in the bias current. This discrepancy exists because our analysis does not account for harmonics in the oscillator waveform. The active devices in an oscillator drive the resonator with a harmonic-rich waveform, which also changes the oscillation frequency [1]. In fact, the oscillation frequency will decrease in order to ensure that the energy is balanced between the inductor and the capacitor $[1,2]$. Consequently, the oscillation frequency is altered due to two effects. When the bias current is large, the frequency variation due to harmonic distortion is dominant, causing the oscillation frequency to drop. 


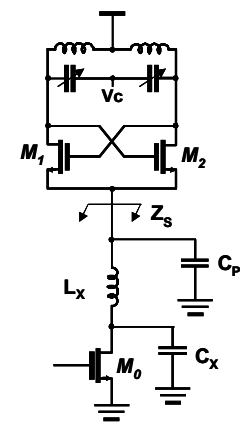

(a)

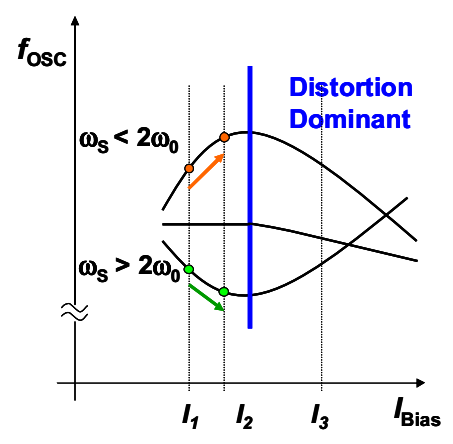

(b)
Fig. 4. (a) Hegazi and Abidi's LC VCO [3]. (b) Relationship between the oscillation frequency and the bias current for the VCO in (a).

\section{OSILLATION FREQUENCY OF HEGAZI and ABIDI'S LC VCO}

In this section, we study the bias current dependence of the oscillation frequency in Hegazi and Abidi's VCO [3], which is shown in Fig. 4 (a).

This VCO is a modified version of the VCO in Fig. 3 (a). A large capacitor with a value of $C_{X}$ (tens of pico farads) is inserted in parallel with $\mathrm{M} 0$ to filter the tail transistor noise. Nevertheless, $C_{X}$ also adds an additional loading on the resonator, as discussed earlier. The quality factor of the additional equivalent inductor is small if $C_{X}$ is large, as suggested by (16).

To address the loading problem, an inductor with a value of $L_{X}$ is inserted between the drain of the tail transistor M0 and the source node of the cross-coupled transistors [3]. Let us define the resonant frequency caused by the inductance $L_{X}$ and the total capacitance at the source node $C_{P}$ to be $\omega_{s}$. If the value of $L_{X}$ is chosen properly such that $\omega_{5}$ is close to the $2^{\text {nd }}$ harmonic frequency of the oscillator, then the source impedance $Z_{S}$ seen by the cross-coupled transistors is nearly infinity at the $2^{\text {nd }}$ harmonic frequency. This can be shown mathematically. Given that $C_{X}$ is much larger than $C_{P}$, the impedance $Z_{S}$ is approximately given by

$$
\left.Z_{S}\right|_{2^{n d} \text { harmonic }}=Z_{2} \approx \frac{j\left(2 \omega_{\text {OSC }}\right) L_{X}}{1-\left(2 \omega_{\text {OSC }}\right)^{2} / \omega_{S}{ }^{2}} \approx \frac{j\left(2 \omega_{0}\right) L_{X}}{1-\left(2 \omega_{0}\right)^{2} / \omega_{S}{ }^{2}}=Z_{20}
$$

where the resonant frequency $\omega_{S}$ is

$$
\omega_{S}=\frac{1}{\sqrt{L_{X} C_{P}}} \approx 2 \omega_{O S C}
$$

As can be seen from (15), when the resonant frequency $\omega_{S}$ is approximately the $2^{\text {nd }}$ harmonic frequency, the impedance $Z_{S}$ approaches infinity.

In order to obtain the oscillation frequency of this VCO, $B_{(-)}$is derived and is given in (17). The oscillation frequency can be then expressed by (18).

$$
\begin{gathered}
B_{(-)} \approx \frac{1-\left(2 \omega_{O S C}\right)^{2} / \omega_{S}{ }^{2}}{4 \omega_{\text {OSC }} L_{X}\left(1+1 / g_{m 2}{ }^{2}\left|Z_{20}\right|^{2}\right)} \\
\omega_{O S C}{ }^{2} \approx \omega_{0}{ }^{2}\left(\left(1-\frac{1}{Q_{L}{ }^{2}}+\frac{1}{Q_{C}{ }^{2}}\right)-\frac{L}{4 L_{X}}\left(1-\frac{\left(2 \omega_{O S C}\right)^{2}}{\omega_{S}{ }^{2}}\right)\left(1-\frac{1}{g_{m 2}{ }^{2}\left|Z_{20}\right|^{2}}\right)\right)
\end{gathered}
$$

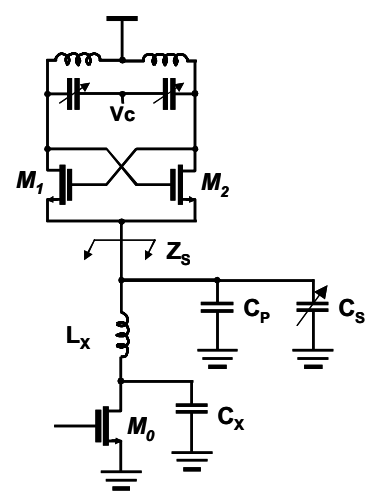

Fig. 5. LC VCO with a digitally controlled capacitor $\mathrm{C}_{\mathrm{S}}[5]$.

Equation (18) shows that the oscillation frequency is again a function of the bias current, through the dependence on $g_{m 2}$.

Another important point to note is that, the oscillation frequency is also affected by the resonant frequency $\omega_{s}$. Depending on the polarity of the term $\left(1-\left(2 \omega_{\text {osc }}\right)^{2} / \omega_{s}^{2}\right)$ the oscillation frequency can either increase or decrease with an increase in the bias current. For example, if $\omega_{5}$ is smaller than $2 \omega_{\text {osc }},\left(1-\left(2 \omega_{o s c}\right)^{2} / \omega_{s}^{2}\right)$ is negative and hence the oscillation frequency will increase with an increase in the bias current. On the other hand, if $\omega_{s}$ is larger than $2 \omega_{\text {osc }}$, $(1-$ $\left.\left(2 \omega_{\text {osc }}\right)^{2} / \omega_{s}^{2}\right)$ is positive and hence the oscillation frequency will decrease with an increase in the bias current. If $\omega_{s}$ is equal to $2 \omega_{o s c}$, the term $\left(1-\left(2 \omega_{o s c}\right)^{2} / \omega_{s}^{2}\right)$ is zero. Thus, the oscillation frequency is independent of the bias current when $\omega_{s}$ is equal to $2 \omega_{\text {osc }}$.

Once again the analysis above is only valid for small bias currents. At large bias currents, distortion effects become important. Simulations show that, when $\omega_{s}$ is smaller than $2 \omega_{\text {osc }}$, the oscillation frequency increases for small bias currents but drops at larger bias currents. Conversely, when $\omega_{s}$ is larger than $2 \omega_{o s c}$, the oscillation frequency drops for small bias currents but can increase at larger bias currents. This relationship between the oscillation frequency of the Hegazi and Abidi's LC VCO and the bias current is shown in Fig. 4 (b).

\section{DIGITAL TRIMMING TECHNIQUE}

Although the Hegazi and Abidi's VCO was able to achieve low phase noise performance [3], two obstacles need to be addressed in practical implementations. First, the VCO only works optimally at a particular oscillation frequency. At other frequencies the $2^{\text {nd }}$ harmonic frequency is altered but the resonant frequency $\omega_{s}$ is unchanged. Simulation results show that the phase noise performance is significantly degraded if the resonant frequency $\omega_{s}$ is different from the $2^{\text {nd }}$ harmonic frequency $2 \omega_{\text {osc. }}$ This observation was also confirmed in [4], where a close-in 
phase noise difference of $10 \mathrm{~dB}$ was measured when the VCO was tuned from $725 \mathrm{MHz}$ to $806 \mathrm{MHz}$ in Hegazi and Abidi's VCO. To reduce this problem, a digitally controlled capacitor $C_{S}$ can be added in parallel with the capacitor $C_{P}$ [5], as shown in Fig. 5. By doing so, it is feasible to change the resonant frequency $\omega_{s}$, and thus track the change of the oscillation frequency.

One remaining obstacle is that the inductance $L_{X}$ and the capacitance $C_{P}$ are very difficult to estimate due to process variations. As a result, it is difficult to achieve the optimal phase noise performance. To resolve this problem, we propose a trimming technique to ensure that the resonant frequency $\omega_{s}$ is equal to the $2^{\text {nd }}$ harmonic frequency of the VCO.

As discussed in Section III, for small bias currents, the oscillation frequency variation due to bias current is dependent on $\omega_{s}$, i.e., the total capacitance $C_{P}+C_{S}$. This observation can be used in our trimming technique. As indicated in Fig. 4(b), bias currents $I_{1}$ and $I_{2}$ are small but $I_{3}$ is large. When the bias current is changed from $I_{1}$ to $I_{2}$, if the oscillation frequency increases, then we know that $\omega_{s}$ is smaller than $2 \omega_{\text {osc }}$. The capacitance $C_{S}$ should be reduced. On the other hand, if the oscillation frequency decreases when the bias current is changed from $I_{1}$ to $I_{2}$, then $\omega_{S}$ is larger than $2 \omega_{\text {osc }}$ and the capacitance $C_{S}$ should be increased. This trimming procedure should be continued until the condition $f_{\text {osc } I_{1}}=\left.f_{\text {osc }}\right|_{I_{2}}$ is met. After the capacitor $C_{S}$ is trimmed, a large bias current, say $I_{3}$, should be used in the VCO to increase the output amplitude for a better phase noise performance.

To demonstrate our proposed trimming technique, we have designed and simulated the LC VCO of Fig. 5 in a $0.18 \mu \mathrm{m}$ CMOS technology with a $1 \mathrm{~V}$ supply voltage. The value of $L_{X}$ is $0.75 \mathrm{nH}$. A 5-bit digitally controlled capacitor $C_{S}$ is used with the least significant bit (LSB) value of $10 \mathrm{fF}$. The simulated oscillation frequency as a function of the bias current for different $C_{S}$ is given in Fig. 6 (only 5 results with different codes for $C_{S}$ are shown). The code of "01110" corresponds to the optimum code, at which the total capacitance $C_{P}+C_{S}$ is resonant with the inductance $L_{X}$ at $2 \omega_{\text {osc. }}$. The codes of " 00100 " and " 01001 " correspond to a smaller $C_{S}$ than the optimum value ( $\omega_{s}$ larger than $\left.2 \omega_{\text {osc }}\right)$ while the codes of "10011" and " 11000 " correspond to a larger $C_{S}$ than the optimum value ( $\omega_{s}$ smaller than $\left.2 \omega_{\text {osc }}\right)$. As shown in Fig. 6, for bias currents from $0.5 \mathrm{~mA}$ to $1.0 \mathrm{~mA}$, the variation of the oscillation frequency behaves the same way as predicted by our analysis.

The simulated phase noise at both $50 \mathrm{kHz}$ offset and $1 \mathrm{MHz}$ offset for different $C_{S}$ is summarized in Fig. 7. A $4 \mathrm{~mA}$ bias current is used for each simulation. When using the optimum code " 01110 " the phase noise is much better than those with other codes. This indicates that our proposed trimming technique can have a significant impact on the overall performance of the oscillator.

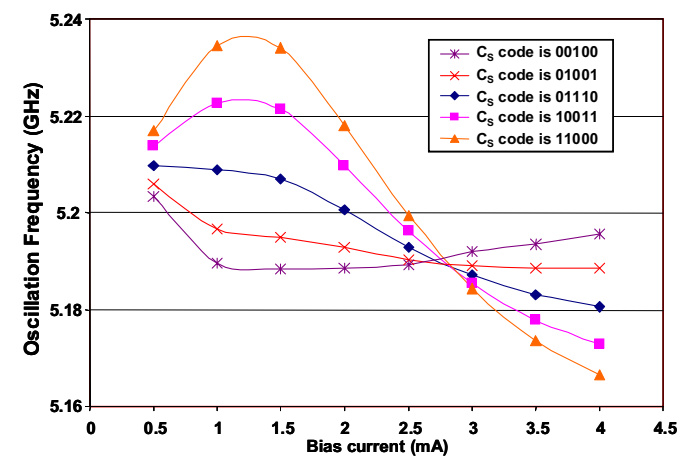

Fig. 6. Simulated oscillation frequency of the VCO in Fig. 5 vs. the bias current for different $\mathrm{C}_{\mathrm{S}}$.

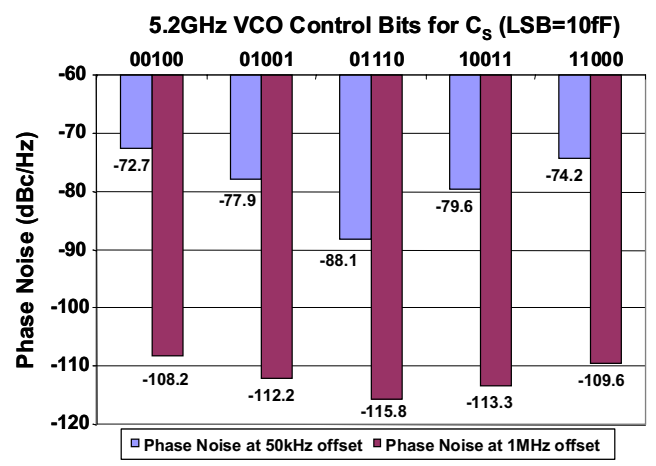

Fig. 7. Simulated phase noise of the $5.2 \mathrm{GHz}$ VCO in Fig. 5 using a $4 \mathrm{~mA}$ bias current for different $\mathrm{C}_{\mathrm{S}}$.

\section{CONCLUSIONS}

In this paper, the bias current dependence of the oscillation frequency of a differential LC VCO is described. Simulation results validate our analysis. With the aid of our analysis, we have proposed a digital trimming technique to optimize phase noise performance of Hegazi and Abidi's LC VCO. An improvement in the phase noise of $3 \mathrm{~dB}$ at $1 \mathrm{MHz}$ offset and $10 \mathrm{~dB}$ at $50 \mathrm{kHz}$ offset can be achieved.

\section{ACKNOWLEDGMENT}

This work is supported by the Semiconductor Research Corporation under contract 2003-HJ-1076.

\section{REFERENCES}

[1] J. Groszkowski, Frequency of Self-Oscillations. Oxford, Pergamon Press, 1964.

[2] J. J. Rael and A. Abidi, "Physical processes of phase noise in differential LC oscillators," in Custom Integrated Circuits Conf., pp. 569572, May 2000.

[3] E. Hegazi, H. Sjoland, and A. Abidi, "A filtering technique to lower oscillator phase noise," in Dig. Tech. Papers IEEE ISSCC, pp. 364-365, Feb. 2001.

[4] K. Hoshino, E. Hegazi, J. Rael, and A. Abidi, “A 1.5V, 1.7mA 700MHz CMOS LC oscillator with no upconverted flicker noise," in Proc. of the European Solid State Circuits Conference, pp. 337-340, Sep. 2001.

[5] E. Hegazi and A. Abidi, "A $17-\mathrm{mW}$ transmitter and frequency synthesizer for $900-\mathrm{MHz}$ GSM fully integrated in $0.35-\mu \mathrm{m} \mathrm{CMOS,"} \mathrm{IEEE}$ Journal of Solid-State Circuits, vol.38, pp. 782-792, May 2003. 